\title{
How all sizes fit together
}

t ow tolerant are crystals? Identical spheres tend simply to pack into a face-centred cubic crystal when compressed, as experiments on colloidal crystals have shown. But for many colloidal systems the particle sizes are not identical - they have some degree of size dispersity. How much disparity can such a system accommodate while still packing in a crystalline manner?

More than was thought, according to a study by Bommineni and colleagues'. They have used hybrid molecular dynamics/Monte Carlo simulations to look at the phases formed by hard spheres with a large dispersivity - that is, with considerable variation in their radii. Such systems crystallize even with up to $19 \%$ size variation, the researchers say, if compressed slowly enough. How do they manage that when the particle sizes are so diverse?

For some time, it was thought that significant polydispersity causes spheres to separate into coexisting close-packed phases in which the sizes are segregated ${ }^{2}$. But that doesn't actually seem to be what happens. For example, one set of experiments on colloidal silica with $14 \%$ polydispersity showed that the particles would crystallize into single phases (coexisting with the liquid) with complex but regular structures ${ }^{3}$. Among these are so-called Laves phases, found in intermetallic compounds of the type $\mathrm{AB}_{2}$ (such as $\mathrm{MgCu}_{2}$ ) with relatively small ratios of atomic radius (up to around 1.67). Laves phases have also been seen in simulations of hard spheres with $12 \%$ dispersity ${ }^{4}$.
In other words, such binary phases seem to offer a 'coping strategy' for achieving close packing with a distribution of particle sizes. Bommineni et al. show that this remains an effective option with a still greater size distribution than in those earlier studies - but that the structural options then become even more diverse and complex too. Finding stable phases in their simulations involves making 'moves' that produce a new configuration of two adjacent particles in each step, which is accepted or rejected with a probability determined by the usual Boltzmann-factor weighting for the Monte Carlo method. The researchers explore two types of polydispersity. In the static case each particle has a fixed size, and a step exchanges two particle positions. But a mixture of particle sizes can also result from dynamic changes - particles getting bigger or smaller, simulated in a given step by random changes in the radii of the two particles that preserve a constant packing fraction.

Both the static and dynamic simulations produce ordered phases over a wide range of dispersity - up to $17 \%$ (after which equilibration is too slow to follow) and 19\% respectively. As well as Laves phases, the spheres can adopt so-called Frank-Kasper (FK) phases, which are also seen for some intermetallic compounds (Laves phases are in fact often regarded as a subset of these). FK structures can have complex unit cells and stoichiometries, or may be non-stoichiometric, and they include non-periodic quasicrystalline phases as well as true crystals. They commonly contain icosahedral

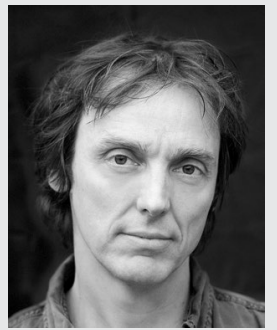

Philip Ball

clusters, and might be considered a kind of transitional realm between simple close-packed crystals and liquids, which also often exhibit icosahedral local order. One phase seen at high dispersity corresponds to the $\mathrm{AB}_{13}$ structure reported in opal gemstones, which are natural colloidal crystals.

Dynamic dispersity seems in fact to assist the formation of these ordered states, so that factors that allow variations in effective particle size slowly decaying, 'soft' interparticle forces, say, or flexible ligands could promote crystallization. More generally the work hints at deep geometric connections between the configurational spaces available to discrete and continuous particle distributions.

Published online: 18 April 2019 https://doi.org/10.1038/s41563-019-0362-z

References

1. Bommineni, P. K., Varela-Rosales, N. R., Klement, M. \& Engel, M. Phys. Rev. Lett. 122, 128005 (2019).

2. Sollich, P. \& Wilding, N. B. Phys. Rev. Lett. 104, 118302 (2010).

3. Cabane, B. et al. Phys. Rev. Lett. 116, 208001 (2016).

4. Lindquist, B. A., Jadrich, R. B. \& Truskett, T. M. J. Chem. Phys. 148, 191101 (2018). 